\title{
ИСКУССТВЕННЫЙ ИНТЕЛЛЕКТ В СИСТЕМАХ КОРПОРАТИВНОГО УПРАВЛЕНИЯ
}

\author{
Турлуев Ризван Рамзанович \\ старший преподаватель
}

Чеченский Государственный университет

\begin{abstract}
Аннотация: Наличие прямой причинно-следственной связи между управленческими решениями и аналитико-исследовательской ролью информации позволяет доказать стратегическую значимость информационных ресурсов. Характерно, что потребность в данных рассматривается на уровне руководящего звена компании или организации. Данная позиция указывает на однозначность подтверждения, касающегося итогов проанализированных данных и возможности их монетизации. Информационные данные представляют собой некое фундаментальное основание, востребованное ключевыми структурными звеньями любой компании. Информация служит эффективным идентификатором потенциальных возможностей и вероятных потерь компании в условиях нестабильности бизнес-среды, а также качественной поддержкой со стороны стратегически значимых инициатив. Немаловажное значение сегодня придаётся возможностям совместного применения актуальной информации со стороны всех подразделений компании, чтобы максимально быстро отреагировать на преобразования экономического пространства. Следует отметить, что информация является своего рода активами, уровень эффективности которых особенно значим для успешной деятельности. В статье рассмотрен искусственный интеллект в системах корпоративного управления.
\end{abstract}

Ключевые слова: Искусственный интеллект, корпоративное управление, эффективность.

1. Введение (Introduction);

Искусственный интеллект в системах корпоративного управления понимается как концепт, описывающий, каким образом планируется и собирается, создаётся и организуется, используется и контролируется, распространяется и удаляется информация. Процесс обеспечивает выявление 


\section{ЦИФРОВИЗАЦИЯ: РОССИЯ И СНГ В КОНТЕКСТЕ ГЛОБАЛЬНОЙ ТРАНСФОРМАЦИИ}

и использование ценных данных/информации с целью поддержки работы компании и увеличения цены бизнес-процессов.

2. Материалы и методы (Materials and Methods)

При написании данной статьи были применены такие методы научного исследования, как изучение научной литературы по теме работы, нормативноправовой базы, аналитический и сравнительный методы.

3. Результаты и обсуждение (Results and Discussion);

Искусственный интеллект в системах корпоративного управления может быть представлен не исключительно управлением сырыми данными, а рассматривается с точки зрения контекстуальных метаданных, дополнительных «данных об информации», с учётом «информации в контексте» («data in context»). Управление информацией представлено анализом и моделированием, управлением и работой с хранилищами, а также извлечением, преобразованием и загрузкой. Кроме прочего, в процессе управления происходит обеспечение качественных характеристик и защита информации, её шифрование и любая работа с метаданными, включая архитектуру. Существует ряд наиважнейших факторов, обеспечивающих максимальную успешность управления. Всем пользователям гарантирован доступ к информации посредством разных каналов, а также ценность эксплуатируемых данных. При этом совместное применение (data sharing) не ограничивается только пределами компании, но и доступно другим организациям, а качественные характеристики сохраняются на достаточном уровне [1].

Немаловажное значение придаётся юридическим требованиям, предъявляемым к безопасности и защите частных информационных сведений, их целостности и конфиденциальности, что позволяет обеспечить максимально высокую эффективность и результативность осуществляемой компанией деятельности по обработке информационных потоков. Моделью информации определяются ключевые сущности и взаимосвязи, предупреждающие избыточность, а также архитектурные ухудшения. Компания может обладать историческими, но уже неиспользуемыми данными, а также информацией, недоступной для потенциальных пользователей [6]. Особого внимания требует информация, предоставляемая широкому кругу людей, недостаточно эффективные и устаревшие способы сбора, сохранения и анализа, применения данных. В некоторых случаях 


\section{ЦИФРОВИЗАЦИЯ: РОССИЯ И СНГ В КОНТЕКСТЕ ГЛОБАЛЬНОЙ ТРАНСФОРМАЦИИ}

компания не способна справляться с получением требуемой информации, снижением качества и потерей целостности.

Необходимо принимать во внимание и тот факт, что не всегда есть реальная возможность понять, действительно ли полученная информация обладает исходными данными достаточно высокого качества. Сложность оценки данных обусловлена исходным отсутствием специальных показателей, требуемых для сравнительного анализа. Например, упомянем низкокачественные данные, полученные в результате плохой проверки при таких процедурах, как ввод и/или проведение обновления, которые в итоге не позволяют сформировать достоверную отчётность. Кроме прочего, немаловажное значение имеет согласованность всей информации, которая генерируется разными оперативными и внутренними системами, формируемыми и применяемыми в процессе деятельности.

В широком понимании слова, на сегодняшний день, управление информацией представляет собой применение большого количества современных технологий, разнообразных стандартов и перспективных процессов. Однако ключевые вопросы, касающиеся качественных характеристик информации (уровень безопасности, соответствие корпоративной этике и положениям нормативных актов, фокусируют внимание на корректности справочной информации и непротиворечивости, метаданным и мастер-данным. В некоторых случаях, при необходимости допускается включение направлений прикладной обработки данных в виде СУБД, ВІ и документооборота. Тем не менее, в настоящее время очень большое количество компаний и предприятий пребывают в состоянии цифрового преобразования, что способствует резкому расширению круга информации, а также её объёмов [3], практикуя метод размещения корпоративной информации на облачных ресурсах, или аренды необходимых данных. В подобных ситуациях наблюдается изменение требований, предъявляемых к управлению данными с ревизией имеющейся информации.

Чтобы максимально правильно понять концепцию описываемых процессов, нужно указать, что к данной категории не относится управление изменениями, набор процедур по чистке, получению, трансформированию и загрузке информации (ETL). Кроме прочего, к этой области не относится управление мастер-данными (MDM), сохранение и проектирование, а также процессы администрирования баз данных. Несмотря на тот факт, что в 


\section{ЦИФРОВИЗАЦИЯ: РОССИЯ И СНГ В КОНТЕКСТЕ ГЛОБАЛЬНОЙ ТРАНСФОРМАЦИИ}

концепцию по управлению корпоративной информацией могут быть включены некоторые блоки работ, феномен, как правило, рассматривается в качестве методологии управления, а не набора отдельных мероприятий. Кроме прочего, подобная ключевая задача программного обеспечения для управления корпоративной информацией представлена обеспечением качественных характеристик данных. Именно по этим причинам максимально полное раскрытие темы программы требует проведения анализа литературных источников в плане обеспечения высококачественных данных[4].

Руководящим составом предприятия должно применяться в работе полное и непротиворечивое представление в отношении организационной деятельности, включая отдельные подразделения и процессы, осуществляемые на современном рынке. Именно таким способом принимаются стратегически зрелые и оперативные, грамотные решения. Многообразие информационных технологий формирует в ИТ секторе так называемый ландшафт современного предприятия, что подразумевает в современных, активно развивающихся компаниях наличие нескольких десятков специальных учётных систем, поставляющих информацию для топ менеджмента. В современных условиях компания, которая работает со значительными объёмами необходимой информации, разнообразными децентрализованными источниками, недостаточной актуальностью и небольшой структурированностью данных, неминуемо сталкивается с невероятной сложностью решения задач по управлению информацией[5].

Устаревшие, некорректные, фрагментировано неполные, разрозненные данные крайне отрицательно влияют на процессы инвестиционной отдачи, что особенно актуально для компаний с существенными вложениями в ИТ обеспечение бизнес проектов. Проблематика качественных характеристик управления информацией и показатели их интеграции ощутимо воздействуют на сферу рентабельности компаний и предприятий [2]. Как правило, подобные моменты корпоративной среды основываются на неоднородности платформы, когда информация объединена в разных форматах, как генерируемая в компании, так и поступающая извне. Указанный аспект обращает внимание на многочисленные независимые пакеты информации с многократно нескоординированными извлечёнными данными, а также множественными вариантами данных и отчётов. Особенно важно изучить разнообразие и сложность структурных качеств используемых данных, а также процедуру 


\section{ЦИФРОВИЗАЦИЯ: РОССИЯ И СНГ В КОНТЕКСТЕ ГЛОБАЛЬНОЙ ТРАНСФОРМАЦИИ}

пользовательского доступа к информации, который из-за вероятной сложности и трудоёмкости может создавать барьеры для операторов, реализующих бизнес процессы.

В настоящее время известно несколько областей менеджмента, которые относятся к действиям в Data/Information Management. Значимая роль принадлежит Management of data resources как инструмента, который всем ресурсам в обязательном порядке обеспечивает известность и назначение специальных ответственных лиц-управленцев, определяет владельцев информации (ownership) и метаданных. Процесс data administration обеспечивает ответственность в отношении выбора принципа информационных потребностей, выстраивается реестр datain ventory и моделируются данные для конкретной компании. К условиям относится возможность выявлять дублирование и недостатки информации, обеспечивать поддержку контента данных data/information content, а также измерять затраты и грамотно, максимально полно определяя информационную ценность. Процедуры Management of data/information technology позволяют управлять ИТ подразделением, поддерживающим информационные системы компании за счет обращения к таким процессам как проектирование и управление базами данных. К Management of information processes относятся бизнеспроцессы, приводящиеся ИТ-услуги ИТ для применения любой информации. С этой точки зрения компании для управления информацией требуется детально рассмотреть такие мероприятия как полностью контролируемое создание и сбор, доступ и модификация, хранение и удаление, архивирования информации [7].

В соответствии с существующей на сегодняшний день практикой best practices, сфера действия процессами управления информации, представлена управлением неструктурированных данных, не присутствующих в стандартных системах (форматы текста, изображения и звука). Именно этими процессами максимально эффективно обеспечиваются сегодня все качественные характеристики этапов жизненного цикла информации, включая сбор предъявляемых требований и непосредственную эксплуатацию с её завершением в условиях уже установленных временных пределов.

4. Выводы (Conclusion);

Безусловно, ключевое внимание в рамках предоставленной работы, сосредотачивается на таком понятии, как значимость искусственного 


\section{ЦИФРОВИЗАЦИЯ: РОССИЯ И СНГ В КОНТЕКСТЕ ГЛОБАЛЬНОЙ ТРАНСФОРМАЦИИ}

интеллекта в системах корпоративного управления. Принимая во внимания всю сегодняшнюю специфику, командами, поддерживающими процессы управления информацией, безусловно, может также обеспечиваться служба поддержки бизнес-данных. В подобной ситуации таким специалистам предоставляется возможность отвечать на вопросы, касающиеся значения, возможностей и форматов применения информации внутри компании.

Именно по этой схеме осуществляется всё управление метаданными, а также понимание и объяснение типовых характеристик внешних данных, необходимых для идеального выполнения требуемых бизнес-процессов. С данной точки зрения крайне важным является понимание того, что в процессе создания и реорганизации рассматриваемых процессов важно продумать возможность повторного применения информации в условиях конкретной компании. Такая задача вполне заслуженно относится в настоящее время к категории важнейших планов с точки зрения максимально грамотного и высокоэффективного управления информацией.

\section{Список литературы}

1. Демченко И.А., Колесникова Н.А. Современные технологии финансового менеджмента в российских компаниях // Экономика и управление в XXI веке: тенденции развития, 2016. С. 125-129.

2. Дик В.В., Староверова О.В., Уринцов А.И. Технологическая и организационная виртуализация предприятия в условиях информационного общества // Ученые записки Крымского федерального университета имени В.И. Вернадского. Экономика и управление, 2016. С. 35-41.

3. Рыманов, А.Ю. Корпоративное управление: Уч. / А.Ю. Рыманов, И.Ю. Бочарова. - М.: Инфра-М, 2015. - 576 с.

4. Buterin V. A next generation smart contract and decentralized application platform. Ethereum White Paper; 2013

5. Davidson S., De Filippi P., Potts J. Disrupting Governance: The New Institutional Economics of Distributed Ledger Technology. SSRN Electronic Journal. 2016. DOI: 10.2139/ssrn.2811995

6. Filippi P., Loveluck B. The invisible politics of Bitcoin: governance crisis of a decentralised infrastructure [Internet]. Internet Policy Review. 2018. URL: 
https://policyreview.info/articles/ analysis/invisible-politics-bitcoin-governancecrisis-decentralised-infrastructure.

7. Palfreyman J. Blockchain for government? 2015. URL: https://www. $\mathrm{ibm}$.com/blogs/insights-on-business/government/blockchain-for-government/ (accessed 11.10.2020).

8. Iansiti M., Lakhani K.R. The truth about blockchain. Harvard Business Review. 2017;95(1):118-127. 